\title{
Sulfur point defects in crystalline and amorphous silicon
}

\section{Citation}

Mo, Yina, Martin Z. Bazant, and Efthimios Kaxiras. 2004. "Sulfur Point Defects in Crystalline and Amorphous Silicon." Physical Review B 70 (20). https://doi.org/10.1103/physrevb.70.205210.

\section{Permanent link}

http://nrs.harvard.edu/urn-3:HUL.InstRepos:41384086

\section{Terms of Use}

This article was downloaded from Harvard University's DASH repository, and is made available under the terms and conditions applicable to Other Posted Material, as set forth at http:// nrs.harvard.edu/urn-3:HUL.InstRepos:dash.current.terms-of-use\#LAA

\section{Share Your Story}

The Harvard community has made this article openly available.

Please share how this access benefits you. Submit a story.

\section{Accessibility}




\title{
Sulfur point defects in crystalline and amorphous silicon
}

\author{
Yina Mo \\ Department of Physics, Harvard University, Cambridge, Massachusetts 02318, USA \\ Martin Z. Bazant \\ Department of Mathematics, Massachusetts Institute of Technology, Cambridge, Massachusetts 02139, USA \\ Efthimios Kaxiras \\ Department of Physics and Division of Engineering and Applied Sciences, Harvard University, Cambridge, Massachusetts 02318, USA
}

(Received 3 April 2004; revised manuscript received 29 June 2004; published 19 November 2004)

\begin{abstract}
We present first-principles calculations for the behavior of sulfur point defects in crystalline and amorphous silicon structures. By introducing the sulfur point defects at various representative positions in the samples, including substitutional and interstitial sites in the crystal and fourfold coordinated or miscoordinated sites (dangling bond and floating bond sites) in the amorphous, we analyze the energetics in detail and determine the most stable structures. Two important conclusions we draw are: (a) in crystalline $\mathrm{Si}$, the $\mathrm{S}$ defects form pairs in which the two $\mathrm{S}$ atoms are energetically bound but not covalently bonded; (b) in amorphous $\mathrm{Si}$, they preferentially occupy threefold coordinated sites, even when the starting configuration has higher coordination (four- or fivefold). The implications of these results for the electronic structure of sulfur-doped Si samples are also analyzed in the context of the present calculations.
\end{abstract}

DOI: 10.1103/PhysRevB.70.205210

PACS number(s): 61.72.Bb, 61.72.Ji, 61.72.Tt

\section{INTRODUCTION}

Silicon is an important technological material on which most of the semiconductor industry is based. Because of its importance, Si has been studied extensively, in particular as far as point defects are concerned, since it is the presence of such defects that render it useful as an electronic device material. The typical point defects involve the substitution of a $\mathrm{Si}$ atom by another element that can act either as an electron acceptor (positive dopant) or an electron donor (negative dopant). The common dopants involve elements that differ in valence from $\mathrm{Si}$ by only one electron, such as the positive dopants $\mathrm{B}$ and $\mathrm{Al}$, or the negative dopants $\mathrm{P}$ and $\mathrm{As}$; in these cases, the dopant atom provides a single carrier of positive (hole) or negative (electron) charge and the ion is located at a regular crystal site. More complicated situations can arise and can be of interest, from a technological as well as a fundamental point of view. One such case is that of group-VI atoms (chalcogens), like oxygen and sulfur, which in principle can provide two negative carriers when substituting for a $\mathrm{Si}$ atom. However, the ability of these elements to form more complex structures with multiple bonds makes their behavior in the host $\mathrm{Si}$ crystal more difficult to understand.

Oxygen is an element that binds strongly to $\mathrm{Si}$ and is abundant in the atmosphere, so it is readily incorporated in a sample. The presence of oxygen atoms in a silicon crystal has received much attention. Single interstitial oxygen atoms are believed to be able to hop between the interstitial sites and oxygen dimers can diffuse even more freely. ${ }^{1-4}$ Sulfur has the same valence with oxygen and is in the same row of the Periodic Table with $\mathrm{Si}$, so its incorporation in the $\mathrm{Si}$ crystal should be also quite favorable. It is natural to expect that substitutional $\mathrm{S}$ would induce less strain than $\mathrm{O}$ in the $\mathrm{Si}$ lattice, having a covalent radius of $1.04 \AA$, compared to silicon's $1.17 \AA$ and to oxygen's $0.66 \AA .^{5}$ The behavior of $\mathrm{S}$ as a point defect in $\mathrm{Si}$ requires further clarification, both from the experimental and the theoretical point of view.

In this paper we study the behavior of sulfur point defects in $\mathrm{Si}$, from the theoretical view point, using first-principles electronic structure calculations based on the density functional theory. Our results show that sulfur indeed behaves in some respects similarly to oxygen, but we also find some new bonding structures that are different from the oxygen doping cases. Previous theoretical work in chalcogen defects in silicon includes several studies similar in approach to what we pursue here ${ }^{6-8}$ Compared to these earlier studies, our calculations provide a much more detailed analysis of the behavior of sulfur atoms in the sample and lead to some unexpected conclusions that have not been found in any earlier work.

In addition to the studies in crystalline $\mathrm{Si}$, we have undertaken theoretical studies of sulfur point defects in amorphous silicon. Amorphous silicon is a material of equal technological interest to crystalline silicon. It has wide applications in phovoltaics, where its electronic structure is of paramount importance.

The theoretical study of amorphous $\mathrm{Si}$ is complicated because there is no unique model structure. Typically, cells with disordered arrangements of atoms are generated by computer simulations, on which periodic or open boundary conditions are imposed. The models for amorphous Si involve coordination defects, that is, atoms that have coordination lower (threefold) or higher (fivefold) than the normal fourfold coordination of covalently bonded $\mathrm{Si}$ atoms. The former type of defect is called a "dangling bond" defect, since the Si atoms are missing one of their regular covalent bonds, while the latter type of defect has been called the "floating bond" defect. ${ }^{9}$ Experiments also support the presence of coordination defects in amorphous $\mathrm{Si}$, although there remains some uncertainty as to the relative abundance of each type of defect. The presence of coordination defects leads to important electronic effects. The disorder of the net- 
work of atoms itself leads to localization of the electron states in amorphous silicon, which can be studied by electronic structure analysis ${ }^{10}$ and identification of the band tail states. ${ }^{11}$ Intrinsic defect states associated with dangling bonds and floating bonds have been contrasted ${ }^{9}$ and analyzed in detail. ${ }^{12-14}$ More recently, the energy landscape of amorphous silicon ${ }^{15}$ and the energetics of hydrogen in amorphous silicon ${ }^{16}$ have also been studied. The samples we have generated and studied involve structures with dangling bond and floating bond defects, as well as structures that have no coordination defects. In all of these, we have introduced sulfur atoms at judiciously chosen substitutional sites and studied in detail their electronic properties.

Another direct motivation for our study of sulfur atoms in crystalline and amorphous silicon samples comes from some recent experiments, ${ }^{17,18}$ which examine optical properties, chemical composition, and structure of silicon microstructures formed by femtosecond and nanosecond laser irradiation in the presence of $\mathrm{SF}_{6}$. In these studies, below-band gap light absorption and photocurrent generation are observed, ${ }^{19,20}$ which can be attributed to sulfur impurities in a microcrystalline surface layer. ${ }^{21}$ In the sample formed by nanosecond laser irradiation, these features persist even after high tempareture annealing, ${ }^{22}$ from which we infer that structures involving stable sulfur defects are formed. These intriguing experimental results raise the question of the nature of electronic states in amorphous $\mathrm{Si}$ and their possible differences or similarities to the corresponding states in crystalline Si.

The rest of this paper is arranged as follows: In Sec. II, we discuss the methodology employed for the first-principles electronic structure calculations, as well as the methods employed to produce and relax the amorphous samples. In Sec. III we give the major results for sulfur point defects in crystalline Si. In Sec. IV we discuss properties of the amorphous $\mathrm{Si}$ which include or do not include intrinsic coordination defects, when sulfur atoms are substitutional or interstitial at representative Si sites. Finally, in Sec. V we give our conclusions from the comparative study of sulfur defects in crystalline and amorphous silicon, and make contact with the recent experimental results of laser irradiation of $\mathrm{S}$-doped $\mathrm{Si}$ samples. We also provide theoretical predictions about the optical properties of S-doped crystalline and amorphous $\mathrm{Si}$ samples, under equilibrium conditions.

\section{METHODOLOGY}

\section{A. First-principles calculations}

In the present paper, first-principles total energy calculations within the density functional theory (DFT) ${ }^{23,24}$ are employed to study the coordination, energy, electronic, and other properties of the pure and sulfur doped silicon samples. The DFT results reported here are based on the local density approximation for the exchange and correlation functional. ${ }^{25,26}$ The calculations are carried out with the totalenergy and molecular-dynamics computer code VASP, ${ }^{27}$ which employs a plane-wave basis. The default plane-wave energy cutoff for sulfur atoms, $197.79 \mathrm{eV}$, from the ultrasoftpseudopotential database,$^{28}$ is used for all calculations. The cell we use to model isolated point defects in crystalline $\mathrm{Si}$ consists of a $3 \times 3 \times 3$ supercell of the conventional cubic cell of bulk Si; this supercell contains 216 atoms. Similar cells were employed for the amorphous samples (see later). Because of the large supercells we used, only the $\Gamma k$-point is used to sample the reciprocal space. The crystal silicon lattice constant determined with these computational parameters is $5.39 \AA$, which compares well to the experimental value of $5.43 \AA$. All atomic relaxations include relaxation of the supercell volume so that volume and symmetry are free to change. For static calculations, Gaussian smearing of width $0.00025 \mathrm{eV}$ is used for the electronic states near the Fermi level. The criterion for convergence of the atomic relaxation was that residual forces on all atoms are smaller in magnitude than $0.01 \mathrm{eV} / \AA$. One of the important properties we study is the coordination changes upon relaxation, especially those induced by the presence of the sulfur impurities. To define coordination, we consider atoms at a distance within $15 \%$ of the regular covalent bond in bulk crystalline $\mathrm{Si}$ to be related by bonds; in our calculations, this cutoff for bonded atoms turns out to be $2.70 \AA$.

\section{B. Preparation of amorphous Si samples}

For a realistic representation of the amorphous structure, a relatively large number of atoms must be included in the cell and the simulation must extend over time scales that allow the structure to relax to a deep energy minimum. Firstprinciples molecular dynamics (MD) simulations are numerically so intensive that they are limited to a short period of time $(\sim 10 \mathrm{ps})$ and a small number of atoms $(<100){ }^{29,30} \mathrm{To}$ overcome this problem, various empirical interatomic potentials for Si have been developed and employed. ${ }^{31}$ Luedtke and Landman ${ }^{32,33}$ tried to produce amorphous silicon structures by rapid quenching of a liquid using an empirical potential developed by Stillinger and Webwer. ${ }^{34}$ Ishimaru, Munetoh and Motooka ${ }^{35}$ generated amorphous networks from melted $\mathrm{Si}$ with various quenching rates by MD simulations employing the Tersoff potential. ${ }^{36}$ Wooten, Winer, and Weaire (WWW) introduced a "bond-switching" method for constructing samples with periodic boundary conditions, in which all atoms are exactly fourfold coordinated without any long range order. ${ }^{37}$ The WWW method is capable of generating amorphous structures with very low strain and defectfree band gaps. ${ }^{38}$ One drawback of this method is that it is not physical, in the sense that the local rearrangements of atoms which lead to the fourfold coordinated amorphous network do not mimic any physical process and could in fact be physically disallowed if the realistic energy cost for such moves were to be considered.

The computer generated models of amorphous $\mathrm{Si}$ are usually judged against experimental results to determine their reliability. The structural, dynamical, and electronic properties of these structures agree reasonably well with experimental results. ${ }^{39-41}$

In this paper, we create samples of bulk amorphous silicon $(a-\mathrm{Si})$ by a standard and physically realistic method, that is, by quenching the liquid phase with explicit molecular dynamics using the environment dependent interaction po- 
tential (EDIP). ${ }^{42}$ We vary the conditions of quenching enough to produce a variety of qualitatively different structural models. We then relax and evaluate these models using first-principles electronic structure methods, which provide a reliable and accurate description of the energetics.

High quality bulk samples of different sizes are prepared with the EDIP using periodic boundary conditions, while allowing the volume to vary at zero pressure. The first step is to create a well-equilibrated liquid by melting a perfect diamond crystal at a very high temperature $(3000 \mathrm{~K})$ for $50 \mathrm{ps}$ and cooling it over $100 \mathrm{ps}$ to $1500 \mathrm{~K}$, where it is equilibrated for another $100 \mathrm{ps}$. Although this is near the bulk melting point, the short time and small system size prevent any nucleation of the crystal phase.

In finite samples in vacuum, surface curvature can further lower the melting point, e.g., to $1370 \mathrm{~K}$ for a nanocrystallite, ${ }^{43}$ but a flat, periodic interface between the liquid and an (already nucleated) crystal remains in equilibrium at the bulk melting point, $T_{c}$. Different, independent measurements have yielded the values, $T_{c}=1500,{ }^{44} 1520,{ }^{45}$ and $1530 \mathrm{~K},{ }^{45}$ which are consistent within simulation error. Note that these values are only $10 \%$ smaller than the experimental melting point of $1685 \mathrm{~K}$, in spite of the fact that the potential was not fit to the melting point or any properties of the liquid. ${ }^{43}$

Next, the sample is gently cooled over $1 \mathrm{~ns}$ to $1000 \mathrm{~K}$ through an abrupt first-order transition to the amorphous state, which occurs over roughly 100 ps near $1200 \mathrm{~K}$ (simulations with flat amorphous-liquid interfaces yield a bulk melting point of $T_{a}=1200^{44}$ or $1170 \mathrm{~K}^{45}$ ). The sample is then annealed at $1000 \mathrm{~K}$ for $2 \mathrm{~ns}$, and cooled to zero temperature over another $2 \mathrm{~ns}$. The sample can be somewhat further purified by heating it gradually back to $1000 \mathrm{~K}$ and repeating the 4 ns annealing/cooling cycle. Since the self-diffusion coefficient at $1000 \mathrm{~K}$ is $0.90 \AA^{2} / \mathrm{ns}$ for the EDIP amorphous $\mathrm{Si}^{44}$ the annealing cycles allow ample time for many structural rearrangements at the scale of the atomic correlation length but insufficient time for diffusion across the entire sample, which would take 100 or 300 ns for $N=64$ or $N$ $=216$, respectively, $N$ being the number of atoms in the supercell.

In these simulations, a subtle and important size effect is observed: Decreasing the sample size (without changing the preparation steps) tends to improve the quality of the resulting amorphous phase, which is presumably due to the reduced relaxation time in smaller systems, perhaps aided by the enhanced effect of the periodic boundary conditions. As previously reported, ${ }^{43}$ large quenched samples $(N>1000)$ tend to have somewhat more than 5\% coordination defects, always fivefolded atoms (floating bonds) in otherwise tetrahedral random network. Here we find that the 216-atom structures prepared according to the steps given above typically possess fewer than ten isolated coordination defects (below 4\%), while a 64-atom structure can be occasionally completely free of defects. We have managed to create such a sample with 64 atoms, which we will take as representative of the ideal, coordination-defect free continuous random network, as it applies to $\mathrm{Si}$; its thermodynamic ${ }^{45}$ and elastic ${ }^{46}$ properties have recently been calculated with EDIP.

We will investigate in detail the properties of two 216atom samples, both of which contain coordination defects (the elastic properties of these samples are reported in Ref. 46). The first sample, denoted as $(a)$, is generated with EDIP by the liquid quench procedure. It has eight floating bond defects $(3.7 \%)$ with otherwise uniform fourfolded coordination. One concern related to the liquid-quench method of sample preparation by molecular dynamics is that, since the liquid has a higher average coordination than the amorphous phase, the resulting defective amorphous structures might have some memory of the liquid state. Although such structures should have physical relevance for ultrarapid laser-melt amorphous $\mathrm{Si}^{47}$ they might not reflect the typical defects found in other forms of amorphous $\mathrm{Si}^{48}$

To overcome these limitations of the liquid-quench simulation approach, we prepared the second amorphous sample by imposing negative pressure and tensile strains, without artificially removing or manipulating any atoms. The sample is derived from an intermediate structure prepared by first applying a negative pressure of -100 GPa to bulk sample $(a)$ at zero temperature, which causes the volume to expand by $10 \%$ through bond stretching, then annealing at $1100 \mathrm{~K}$ for $2 \mathrm{~ns}$ and then slowly cooling back to zero temperature, all the while maintaining constant volume to prevent collapsing back to the original structure, and finally relaxing the volume at zero temperature until the sample returns to zero pressure. During the annealing step, atomic-scale voids (i.e., empty spherical cavities corresponding to at least two atomic volumes) are formed spontaneously to reduce the negative pressure caused by the enlarged volume. During the cooling step these tiny voids are quenched into the structure, which remains enlarged by roughly $10 \%$, even after relaxed to zero pressure at zero temperature. Starting from this intermediate structure, the second amorphous Si sample with coordination defects, denoted as $(b)$, is created by annealing the structure at $1100 \mathrm{~K}$ for $2 \mathrm{~ns}$ and then cooling, all at zero pressure. During the annealing step, the atoms are sufficiently mobile to annihilate all of the voids (thus reducing the excess volume and tensile strain), but there is not enough time for a complete relaxation of the volume. Sample $(b)$ possesses a different set of coordination defects: six floating bonds and one dangling bond, which give a defect density of $3.2 \%$. These simulations show that the structure of amorphous $\mathrm{Si}$ can depend on the sample preparation history, as observed experimentally. ${ }^{48}$

First-principles models of defective amorphous silicon are obtained from the 216-atom empirical bulk samples described earlier by complete structural relaxation, with the same computational parameters as for the crystal calculations. It turns out that the first-principles relaxations significantly changed the features of the empirically created samples; the coordination defects and energy per atom for the relaxed samples are given in Table I. We call these relaxed samples A [obtained from the empirically created sample $(a)$ ] and B [from sample $(b)$ ]. Both have a total of six coordination defects (density 2.8\%). These two samples have an energy per atom which is only slightly above that of the defect-free sample, by $0.135 \mathrm{eV} /$ atom for $\mathrm{A}$ and $0.160 \mathrm{eV} /$ atom for B higher than bulk crystalline Si. These values are consistent with experimental expectations from bulk amorphous energy loss measurements. Also, a volume 
TABLE I. Energetic features and coordination defect populations in the amorphous samples labeled A, B, and C: $N_{F}$ and $N_{D}$ denote the number of floating bonds and dangling bonds, respectively. $\Delta E_{0}$ is the energy difference between each sample and bulk crystalline $\mathrm{Si}$, in eV/atom. $\Delta v / v_{0}$ is the percentage of volume change per atom compared to volume per atom in bulk crystalline Si.

\begin{tabular}{ccccc}
\hline \hline & & & & $\Delta v / v_{0}$ \\
Sample & $N_{F}$ & $N_{D}$ & $\Delta E_{0}(\mathrm{eV} /$ atom $)$ & 1.0 \\
\hline $\mathrm{A}$ & 5 & 1 & 0.135 & 1.0 \\
$\mathrm{~B}$ & 3 & 3 & 0.160 & 2.7 \\
$\mathrm{C}$ & 0 & 0 & 0.132 & \\
\hline \hline
\end{tabular}

increase of about $1.0 \%$ relative to the crystal fits well with the experimental data.

In addition, we will consider a coordination-defect-free sample, based on a 64-atom cell, for which such a structure is possible as mentioned earlier. In order to have a larger sample without coordination defects which is not too different in size from the two 216-atom samples that include coordination defects (so that the dopant density in the two types of samples is comparable), we constructed a 128-atom sample by repeating two 64-atom defect-free cells along one direction and relaxing the structure again with the firstprinciples approach. We refer to this as sample C. All atoms in it are fourfold coordinated with bond lengths ranging from 2.27 to $2.49 \AA$. Its energy is $0.132 \mathrm{eV} /$ atom higher than the energy of bulk crystalline $\mathrm{Si}$, that is, it is the lowest-energy amorphous sample, consistent with the absence of coordination defects in it.

\section{SULFUR DEFECTS IN CRYSTALLINE SI}

For the study of electronic states related to the presence of $\mathrm{S}$ dopants, we considered situations with the $\mathrm{S}$ atoms at substitutional lattice positions or at interstitial positions. The substitutional position is unique and will be denoted as $S_{S}$.

We considered a total of four different interstitial positions for the single $\mathrm{S}$ atom: the first three were highsymmetry positions that are typical of other interstitial atoms; they include the bond-center position, denoted as $\mathrm{S}_{\mathrm{BCI}}$, the hexagonal interstitial position which is situated at the geometric center of a hexagonal ring of $\mathrm{Si}$ atoms, denoted as $\mathrm{S}_{\mathrm{HI}}$, and the tetrahedral interstitial position which is situated at the geometric center of a terahedron defined by four $\mathrm{Si}$ atoms, denoted as $\mathrm{S}_{\mathrm{TI}}$. Our calculations show that the hexagonal or tetrahedral interstitial positions are higher in energy by 1.0 or $1.8 \mathrm{eV}$, respectively, than the bond-center position. Accordingly, in the following we do not provide any more detailed discussions of these two cases. A fourth position for the interstitial $\mathrm{S}$ atoms, which turned out to be the lowest-energy one and is denoted as $\mathrm{S}_{\mathrm{I}}$, corresponds to placing the $\mathrm{S}$ atom on a plane that bisects a $\mathrm{Si}-\mathrm{Si}$ bond, but not along the line joining the two Si atoms. When this structure is allowed to relax, the $\mathrm{S}$ atom assumes a buckled configuration, in which it forms two bonds to Si atoms; this is actually similar to the preferred configuration of an interstitial $\mathrm{O}$ atom. ${ }^{1}$ To verify the stability of the lowest energy configuration for the interstitial, $S_{\text {I }}$, we chose two other sites on the plane bisecting the $\mathrm{Si}-\mathrm{Si}$ line along a bond and after relaxation we obtained a configuration with exactly the same geometric features and energy.

These calculations reveal that for an isolated $\mathrm{S}$ atom, the lowest energy configuration is the substitutional site. The tetrahedral interstitial site, $\mathrm{S}_{\mathrm{TI}}$, which has been considered before as a likely site ${ }^{8}$ has a formation energy that is more than $3 \mathrm{eV}$ higher than the substitutional case. Both these results agree well with earlier theoretical resutls. ${ }^{8}$ The lowest energy configuration that we find for the interstitial, $\mathrm{S}_{\mathrm{I}}$, even though slightly higher than the energy of the substitutional site (by $0.3 \mathrm{eV}$ ), is worth further consideration. First, it is intriguing that the energy of this configuration is $0.95 \mathrm{eV}$ lower than the bond-center interstitial configuration, $\mathrm{S}_{\mathrm{BCI}}$. The big energy difference between $S_{I}$ and $S_{B C I}$ is very different from the $\mathrm{O}$ case, in which these two configurations are almost degenerate in energy. Of course, there are also structural differences in the two off-center configurations for the $S$ and the $\mathrm{O}$ interstitial atoms: specifically, in $\mathrm{S}_{\mathrm{I}}$ we find a buckling angle of $122^{\circ}$ for $\mathrm{Si}-\mathrm{S}-\mathrm{Si}$, much smaller than the $\mathrm{Si}-\mathrm{O}-\mathrm{Si}$ buckling angle, which is about $160^{\circ}$; this can be attributed to the larger atomic size of the $\mathrm{S}$ atoms. In the buckled configuration, the $\mathrm{S}$ atom has two $\mathrm{S}-\mathrm{Si}$ bonds at $2.11 \AA$ each, slighlty longer than in the bond-center configuration, $\mathrm{S}_{\mathrm{BCI}}$, at $2.07 \AA$. Second, the $\mathrm{S}$ atom is considerably farther away from the geometric bond center than the $\mathrm{O}$ atom. As a result of this relaxation, the $\mathrm{S}$ atom has weak interactions with other Si atoms, although it is not covalently bonded to them. This may contribute to the big energy difference between $S_{B C I}$ and $S_{I}$ configurations for $S$, in contrast to the $\mathrm{O}$ case.

For a pair of $\mathrm{S}$ atoms in the crystalline supercell, we considered five different situations.

(a) The two $\mathrm{S}$ atoms taking the place of two adjacent $\mathrm{Si}$ atoms, denoted as $\mathrm{S}_{2 \mathrm{~S}}$ and shown in Fig. 1(a).

(b) One $\mathrm{S}$ atom at the bond center between two $\mathrm{Si}$ atoms and the second $\mathrm{S}$ atom at the closest center of two second nearest neighbored $\mathrm{Si}$ atoms, denoted as $\mathrm{S}_{\mathrm{I}-\mathrm{NI}}$ and shown in Fig. 1(b).

(c) Two $\mathrm{S}$ atoms at neighboring bond centers between $\mathrm{Si}$ atoms, denoted as $\mathrm{S}_{2 \mathrm{I}}$ and shown in Fig. 1(c).

(d) We created a vacancy in the host Si lattice and placed the two $\mathrm{S}$ atoms in the middle of two edges of the tetrahedron centered at the vacancy site, denoted as $\mathrm{S}_{2 \mathrm{I}-\mathrm{V}}$ and shown in Fig. 1(d).

(e) The two substitutional sites within the supercell that are farthest away.

The last situation corresponds energetically to two isolated $\mathrm{S}$ substitutional sites (it has the same formation energy per $\mathrm{S}$ atom as the single $\mathrm{S}$ atom in the supercell). Of the other configurations, the one with the lowest energy is $S_{2 S}$, while $S_{\text {I-NI }}$ and $S_{2 I}$ are about $0.9 \mathrm{eV}$ per $\mathrm{S}$ atom higher in energy than it. Table II summarizes the results for these calculations.

One interesting consequence of these results is that $\mathrm{S}$ atoms in bulk Si bind in pairs to form the pair at nearest neighbor substitutional sites, which lowers the energy by $0.76 \mathrm{eV}$ per $\mathrm{S}$ atom (1.53 eV for the pair), compared to isolated sub- 


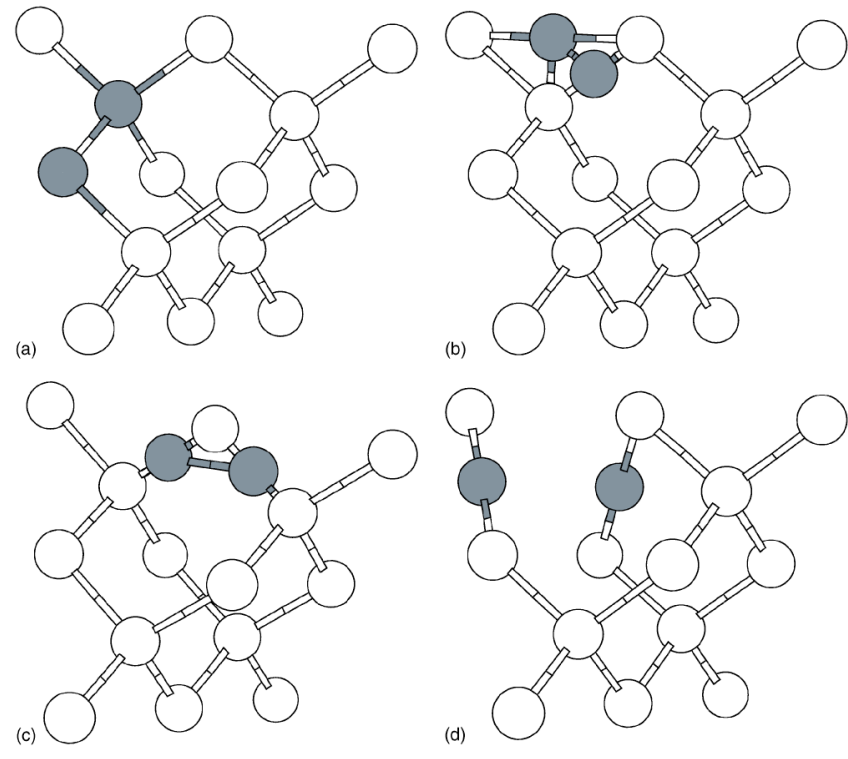

FIG. 1. Configurations considered for two $\mathrm{S}$ atoms in crystalline $\mathrm{Si}$ (the atomic relaxation is not shown): (a) at adjacent substitutional sites, $S_{2 S}$, (b) at a bond center and an adjacent center of two second neighbor $\mathrm{Si}$ sites, $\mathrm{S}_{\mathrm{I}-\mathrm{NI}}$, (c) at two neighboring bond centers, $\mathrm{S}_{2 \mathrm{I}}$, (d) at the centers of opposite edges of a tetrahedron centered at a vacancy site, $\mathrm{S}_{2 \mathrm{I}-\mathrm{V}}$ (see text for details). The grey spheres represent sulfur atoms and the white spheres silicon atoms.

stitutional $\mathrm{S}$ atoms. The energy of configuration $\mathrm{S}_{2 \mathrm{I}-\mathrm{V}}$, which involves the formation of a Si vacancy, includes the vacancy formation energy cost, calculated to be $3.60 \mathrm{eV}$ (this compares favorably with experimental results ${ }^{49}$ ). If this energy cost were not included, the formation energy of this configuration would be $-5.57 \mathrm{eV}$ per $\mathrm{S}$ atom, which is larger than the formation energy of any of the other configurations that do not involve simultaneous creation of lattice defects like the vacancy. In other words, in a Si crystal where vacancies are abundant, the preferred configuration would be $S_{2 I-V}$. However, this would not represent a thermodynamic equilibrium configuration, but a metastable state that can be formed if the vacancy concentration is prohibited from reaching equilibrium, by manipulating for instance the surface of the sample.
All structures containing $S$ atoms have energy gaps that are comparable with that of the bulk. We note that these values are approximately half of the experimental value, a well known deficiency of the theoretical framework employed here. ${ }^{50,51}$ Except for cases $S_{I}, S_{B C I}$, and $S_{2 I-V}$, in which the $\mathrm{S}$ atoms are twofold coordinated, all other $\mathrm{S}$ atoms introduce deep and filled defect states into the energy gap.

The $\mathrm{S}$ atoms at substitutional sites cause distortions, coordination changes and charge redistributions to their local environment, which are shown in Figs. 2 and 3. Some interesting features appear in the configurations involving two $\mathrm{S}$ atoms. Although the $\mathrm{S}$ atoms are energetically bound at nearest-neighbor substitutional sites, they do not actually form a chemical bond among themselves. Instead, there is a geometric repulsion between them: in configuration $S_{2 S}$, the $\mathrm{S}-\mathrm{S}$ distance is $3.07 \AA$, compared to the bond length of $2.33 \AA$ in bulk Si. Both of the S atoms are threefold coordinated. Similarly, in configuration $\mathrm{S}_{2 \mathrm{I}-\mathrm{V}}$, which is the next lowest-energy configuration for the pair of $S$ atoms, the $\mathrm{S}-\mathrm{S}$ distance after relaxation is $2.85 \AA$, compared to $2.69 \AA$, the initial distance when the $S$ atoms are placed at the midpoint sites between pairs of $\mathrm{Si}$ atoms adjacent to the vacancy site. In both cases the $\mathrm{S}$ atoms are optimally twofold coordinated. The electronic charge density distributions shown in Fig. 3 confirm that there is no covalent bonding between the $\mathrm{S}$ atoms, since there is no accumulation of valence charge in the region between the atoms, characteristic of a bond. In connection to the low energies of these configurations, we conclude that the $\mathrm{S}$ atoms always prefer a lower coordination number (two is the optimal one). However, under the strong restriction of the Si crystal structure, they are unable to change their initial coordination by breaking any of the $\mathrm{Si}-\mathrm{S}$ bonds. So they take other opportunities of repelling each other while causing minimal disorder to the crystal Si to lower the total energies.

\section{SULFUR DEFECTS IN AMORPHOUS SI}

In order to study the nature of doping of amorphous Si by $\mathrm{S}$ impurities, and to compare and contrast this to the behavior of crystalline $\mathrm{Si}$, we introduced substitutional $\mathrm{S}$ atoms into the $\mathrm{A}$ and $\mathrm{B}$ amorphous $\mathrm{Si}$ samples and substitutional and

TABLE II. Energetics, structural, and electronic features of configurations with S impurities in crystalline Si. $E_{f}$ is the formation energy (in $\mathrm{eV}$ ) per $\mathrm{S}$ atom, assuming the reservoirs to be bulk $\mathrm{Si}$ and isolated $\mathrm{S}$ atoms. $\Delta V / v_{0}$ is the change of the total volume of the sample upon introduction of the dopant, normalized to the atomic volume $v_{0}$ in bulk crystalline $\mathrm{Si}\left(v_{0}=19.574 \AA^{3}\right) . \varepsilon_{\text {gap }}$ is the energy gap (in $\mathrm{eV}$ ). $\varepsilon_{d}$ and $\varepsilon_{F}$ are the defect state level and the Fermi level relative to the valence band maximum (in eV). For crystalline $\mathrm{Si}$, $\varepsilon_{\text {gap }}=0.57$ and $\varepsilon_{F}=0.00 \mathrm{eV}$.

\begin{tabular}{cccccccc}
\hline \hline & $E_{f}$ & $\Delta V / v_{0}$ & $\varepsilon_{\text {gap }}$ & $\varepsilon_{d}$ & $\varepsilon_{F}$ & $C_{f}$ & Symmetry \\
\hline $\mathrm{S}_{\mathrm{S}}$ & -3.93 & -0.08 & 0.56 & 0.24 & 0.25 & 4 & $T_{d}$ \\
$\mathrm{~S}_{2 \mathrm{~S}}$ & -4.69 & -0.15 & 0.58 & 0.35 & 0.37 & 3,3 & $C_{3 v}$ \\
$\mathrm{~S}_{\mathrm{I}}$ & -3.63 & 0.91 & 0.56 & 0.51 & 0.02 & 2 & $C_{1 h}$ \\
$\mathrm{~S}_{\mathrm{BCI}}$ & -2.68 & 0.47 & 0.55 & 0.49 & 0.05 & 2 & $C_{3 v}$ \\
$\mathrm{~S}_{\mathrm{HI}}$ & -1.78 & 0.61 & 0.54 & 0.35 & 0.35 & 6 & $C_{1 h}$ \\
$\mathrm{~S}_{\mathrm{TI}}$ & -0.87 & 0.79 & 0.61 & 0.14 & 0.14 & 4 & $T_{d}$ \\
$\mathrm{~S}_{2 \mathrm{I}-\mathrm{V}}$ & -3.76 & 0.42 & 0.58 & $0.42,0.57$ & 0.02 & 2,2 & $C_{1 h}$ \\
\hline \hline
\end{tabular}




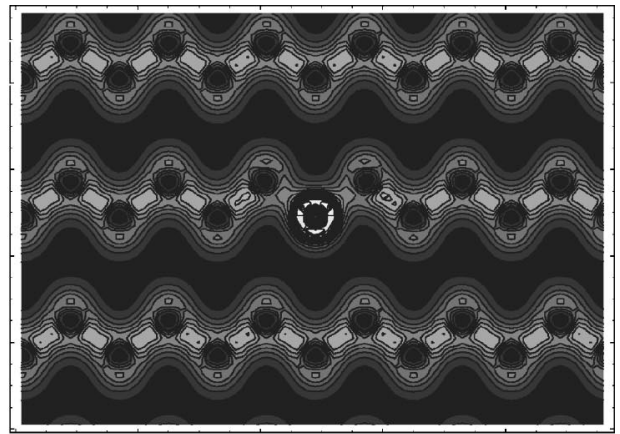

(a)
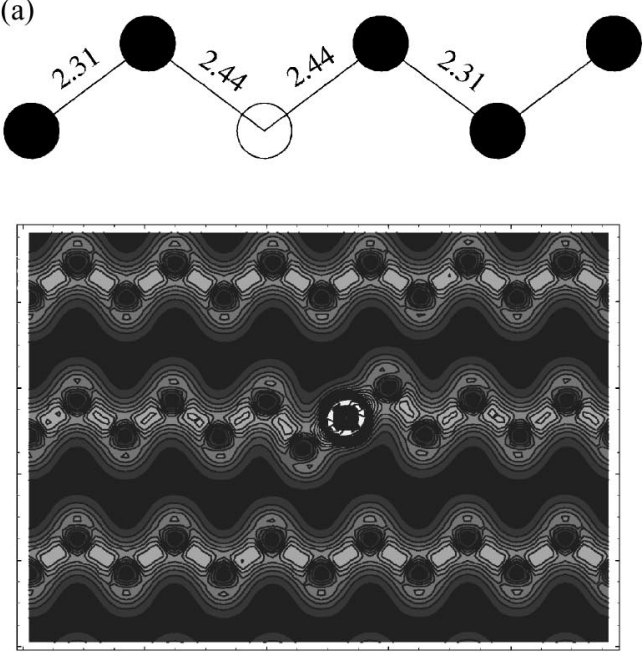

(b)

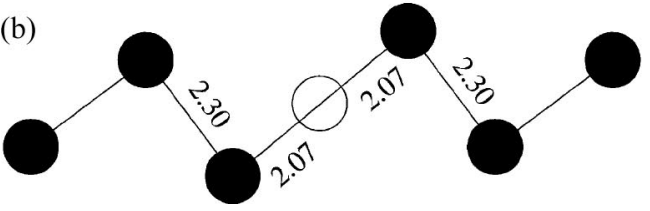

FIG. 2. Geometric structures and electronic charge densities of configurations (a) $\mathrm{S}_{\mathrm{S}}$ and (b) $\mathrm{S}_{\mathrm{I}}$ including atomic relaxation, on the (110) crystal plane. Filled circles represent $\mathrm{Si}$ atoms and open circles represent $\mathrm{S}$ atoms.

interstitial $\mathrm{S}$ atoms into the $\mathrm{C}$ sample. The $\mathrm{S}$ atoms were placed at each one of the coordination defect sites, as well as at certain fourfolded sites that are either very close to the defects or with a relatively long or relatively short average bond length. The energetic and geometric features of these structures are given in Table III.

From Table III, we see that the lowest formation energy corresponds to substitution of dangling bond defect sites. What is striking from this comparison is that a low formation energy is associated with low final coordination for the $\mathrm{S}$ atom (typically threefold, and in one case even twofold coordination), accompanied by a relatively short average bond length, in the range of $2.25 \AA$. Threefold coordination for the $\mathrm{S}$ atom is of course expected when it substitutes a threefold coordinated $\mathrm{Si}$ atom [cases $\mathrm{D}_{1}^{(\mathrm{B})}, \mathrm{D}_{2}^{(\mathrm{B})}, \mathrm{D}_{3}^{(\mathrm{B})}$ ], but it is also found in cases when it substitutes a fourfold [cases $\mathrm{T}_{1}^{(\mathrm{A})}$, $\left.\mathrm{T}_{2}^{(\mathrm{A})}, \mathrm{T}_{5}^{(\mathrm{B})}\right]$, or even a fivefold coordinated $\mathrm{Si}$ atom [cases $\left.\mathrm{F}_{1}^{(\mathrm{B})}, \mathrm{F}_{2}^{(\mathrm{B})}, \mathrm{F}_{3}^{(\mathrm{A})} \mathrm{F}_{4}^{(\mathrm{A})}, \mathrm{F}_{6}^{(\mathrm{A})}, \mathrm{F}_{8}^{(\mathrm{A})}\right]$. In all these cases, when the average bond length is in the neighborhood of 2.20-2.25

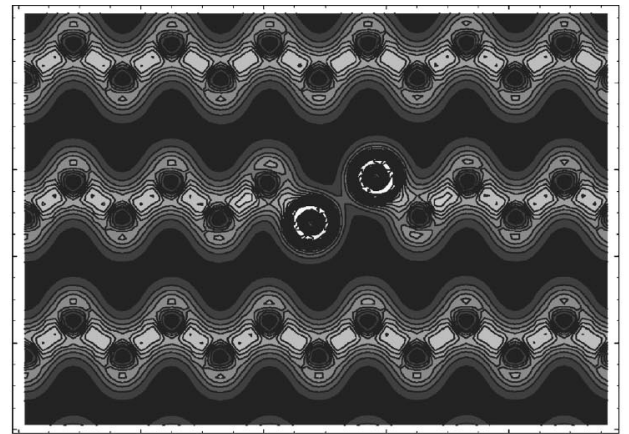

(a)
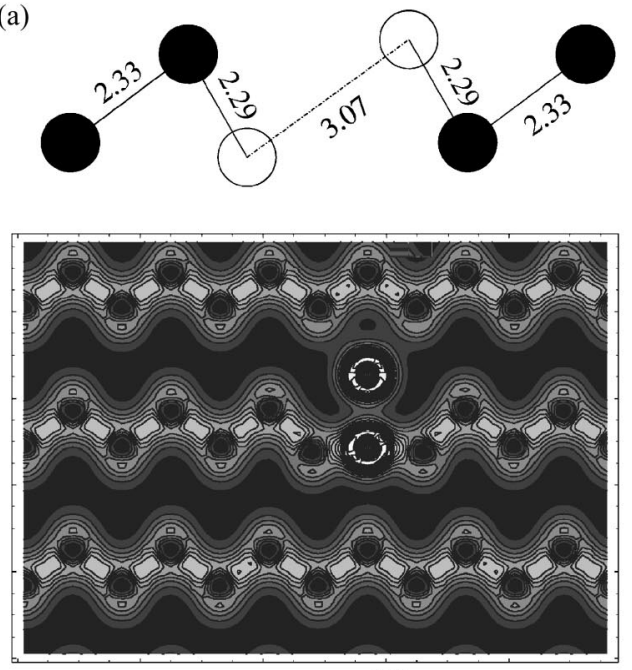

(b)

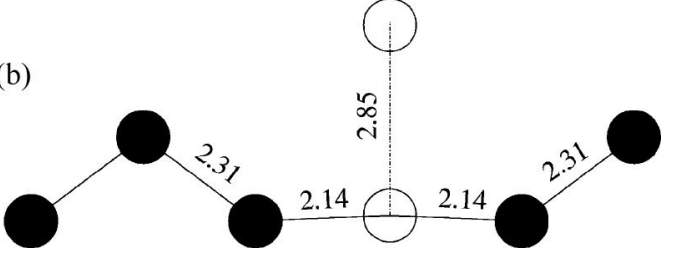
$\mathrm{S}_{2 \mathrm{I}-\mathrm{V}}$.

FIG. 3. Same as in Fig. 2 for the configurations: (a) $\mathrm{S}_{2 \mathrm{~S}}$ and (b)

and the formation energies are lower than $-5.20 \mathrm{eV}$; notice that the sum of covalent radii for $\mathrm{Si}$ and $\mathrm{S}$ is $2.21 \AA$. In contrast to this, when the average bond length exceeds $2.30 \AA$ the formation energy is around $-5 \mathrm{eV}$ or higher. The only exception to this general trend is case $\mathrm{T}_{1}^{(\mathrm{A})}$, which has threefold final coordination and low formation energy, but relatively long average bond at $2.32 \AA$. The fact that the trend is not obeyed in all cases is a reflection of the diversity of local environments in amorphous $\mathrm{Si}$; specifically, the local environment of a particular site may not make it feasible for the $\mathrm{S}$ dopant atom to achieve both its preferred coordination and its optimal bond length. This is probably the situation in case $\mathrm{T}_{1}^{(\mathrm{A})}$.

What is surprising about the tendency of S atoms to form threefold coordination, especially in the cases when the original configuration had fourfold or fivefold coordination, is that covalent bonds are eliminated (at least one in each case). In case $\mathrm{D}_{4}^{(\mathrm{A})}$, where the final configuration involves a twofold coordinated S atom, one covalent bond is eliminated relative to the initial configuration and the formation energy is also very low $(-6.06 \mathrm{eV})$. The important thing to notice is 
TABLE III. Energetic and geometric features of S point defects in amorphous $\mathrm{Si}$ samples. D, F and $\mathrm{T}$ stand for dangling-bond, floating-bond, and tetrahedral sites, where the substitutional $\mathrm{S}$ atoms are introduced; the superscript in each case denotes the sample (A, B, and $\mathrm{C}$, as described in the text). $E_{f}$ (given in $\mathrm{eV}$ ) is the formation energy, taking the ideal $a$-Si sample $\mathrm{C}$ as the reservior for the $\mathrm{Si}$ atoms and the isolated $\mathrm{S}$ atoms for the $\mathrm{S}$ reservior. The different cases are ordered in increasing formation energy. $\Delta V / v_{0}$ is the change in total volume of the sample upon the substitution, normalized to the crystalline atomic volume $v_{0}$. $C_{f}$ is the final coordination of the $\mathrm{S}$ atom (the intitial coordination of the $\mathrm{Si}$ atom being determined by the site identity, $\mathrm{D}, \mathrm{F}$, or T) and $\bar{b}_{i}, \bar{b}_{f}$ are the initial and final average bond lengths of the site.

\begin{tabular}{lrrrrr}
\hline \hline Site & $E_{f}$ & $\Delta V / v_{0}$ & $\bar{b}_{i}$ & $C_{f}$ & $\bar{b}_{f}$ \\
\hline $\mathrm{D}_{1}^{(\mathrm{B})}$ & -6.55 & 0.41 & 2.31 & 3 & 2.24 \\
$\mathrm{D}_{2}^{(\mathrm{B})}$ & -6.29 & 0.55 & 2.33 & 3 & 2.26 \\
$\mathrm{D}_{3}^{(\mathrm{B})}$ & -6.25 & 0.36 & 2.32 & 3 & 2.27 \\
$\mathrm{D}_{4}^{(\mathrm{A})}$ & -6.06 & -0.08 & 2.41 & 2 & 2.17 \\
\hline $\mathrm{F}_{1}^{(\mathrm{B})}$ & -5.96 & 0.55 & 2.48 & 3 & 2.27 \\
$\mathrm{~F}_{2}^{(\mathrm{B})}$ & -5.30 & 0.25 & 2.45 & 3 & 2.25 \\
$\mathrm{~F}_{3}^{(\mathrm{A})}$ & -5.26 & -0.36 & 2.42 & 3 & 2.24 \\
$\mathrm{~F}_{4}^{(\mathrm{A})}$ & -4.99 & -0.44 & 2.45 & 3 & 2.35 \\
$\mathrm{~F}_{5}^{(\mathrm{B})}$ & -4.95 & 0.20 & 2.45 & 4 & 2.34 \\
$\mathrm{~F}_{6}^{(\mathrm{A})}$ & -4.83 & -0.26 & 2.46 & 3 & 2.32 \\
$\mathrm{~F}_{7}^{(\mathrm{A})}$ & -4.34 & -0.44 & 2.42 & 4 & 2.39 \\
$\mathrm{~F}_{8}^{(\mathrm{A})}$ & -4.05 & -0.04 & 2.51 & 3 & 2.39 \\
\hline $\mathrm{T}_{1}^{(\mathrm{A})}$ & -5.81 & -0.25 & 2.43 & 3 & 2.32 \\
$\mathrm{~T}_{2}^{(\mathrm{A})}$ & -5.50 & -0.05 & 2.39 & 3 & 2.26 \\
$\mathrm{~T}_{3}^{(\mathrm{B})}$ & -5.09 & 0.17 & 2.28 & 4 & 2.33 \\
$\mathrm{~T}_{4}^{(\mathrm{A})}$ & -4.89 & -0.58 & 2.28 & 4 & 2.30 \\
$\mathrm{~T}_{5}^{(\mathrm{B})}$ & -4.63 & 0.49 & 2.42 & 3 & 2.35 \\
\hline \hline & & & & & \\
\hline
\end{tabular}

that although the formation energy of $\mathrm{D}_{4}^{(\mathrm{A})}$ is higher than the three dangling-bond-site $\mathrm{S}$ substitutions in sample $\mathrm{B}$, it has a total energy about $5 \mathrm{eV}$ lower than that of any of the three cases in sample B because sample A has a total energy that is $5.46 \mathrm{eV}$ lower than that of sample B. In other words, when a $\mathrm{S}$ atom goes into an $a$-Si sample and the system is well equilibrated (e.g., by further annealing), configuration $\mathrm{D}_{4}^{(\mathrm{A})}$ would be most preferred.

The striking preference of S atom to form threefold coordinated structures, even when starting from a floating bond or a fourfold coordinated site, should have important implications in the electronic behavior of this dopant as well. Unfortunately, in the amorphous samples with several coordination defects there is no gap even in the undoped case, so it is rather difficult to decipher the effect of the dopant from that of other defects, especially since the S dopant leads to significant atomic relaxation which is accompanied by shifts in many electronic states. For these reasons, we have not attempted to examine in more detail the electronic structure of the doped amorphous samples A and B. Instead, in order to understand better the electronic effects of $\mathrm{S}$ doping in amor- phous $\mathrm{Si}$, we turn our attention to sample $\mathrm{C}$, which has only fourfold coordinated sites to begin with, but also has a clear gap in the electronic spectrum.

In sample $\mathrm{C}$, we chose eight different substitutional sites to introduce the $\mathrm{S}$ dopant atom: one with the longest average bond length, referred to as $\mathrm{T}_{6}^{(\mathrm{C})}$, one with the shortest average bond length, referred to as $\mathrm{T}_{13}^{(\mathrm{C})}$, and six with their average bond length very close to the average bond length in the entire sample, listed from $\mathrm{T}_{7}^{(\mathrm{C})}$ to $\mathrm{T}_{12}^{(\mathrm{C})}$. Another three sites were chosen, in which the $S$ atoms are placed in the middle of relatively long $\mathrm{Si}-\mathrm{Si}$ bonds, referred to as $\mathrm{I}_{1}^{(\mathrm{C})}, \mathrm{I}_{2}^{(\mathrm{C})}$, and $\mathrm{I}_{3}^{(\mathrm{C})}$. The nature of these sites has a rough analogy to the interstitial sites in crystalline $\mathrm{Si}$, although the nature of the amorphous network is such that it makes the term interstitial rather ill defined. Nevertheless, we employ this term here to make the comparison more direct. In Table IV we include the results of these calculations, in what concerns both energetics and geometric arrangments, as well as electronic structure features.

Of all the cases in sample $\mathrm{C}$, the interstitial $\mathrm{S}$ atoms have the lowest formation energies. Their identified defect states are empty and close to the bottom of the conduction bands [in one case, $\mathrm{I}_{1}^{(\mathrm{C})}$, the defect state is below the conduction band minimum, inside the gap]. The three sites we tried have relatively long bond lengths that are between 2.40 and $2.50 \AA$; there are fewer than $10 \%$ such bonds in the entire sample. Apparently, the amorphous network has enough structural flexibility to accommodate these additional $\mathrm{S}$ atoms without major coordination changes farther from the defect site, with the adjacent $\mathrm{Si}$ atoms remaining fourfold coordinated. As a check, we also considered another interstitial site, at the center of a $\mathrm{Si}-\mathrm{Si}$ bond with a relatively short bond length. It turns out that the local structure in this case changed significantly, and the $\mathrm{S}$ atom ended up threefold coordinated with a much higher formation energy.

Of all the substitutional cases, except for cases $\mathrm{T}_{6}^{(\mathrm{C})}$ and $\mathrm{T}_{13}^{(\mathrm{C})}$, all other $\mathrm{S}$ atoms end up with threefolded coordination. In fact, case $\mathrm{T}_{13}^{(\mathrm{C})}$ is not much different because, even though by our strict definition of coordination it has four nearest neighbors, three of those are at distances around $2.25 \AA$ and the fourth is much farther away at $2.49 \AA$. In case $\mathrm{T}_{6}^{(\mathrm{C})}$, the $\mathrm{S}$ atom is twofold coordinated, having broken two of the bonds that the $\mathrm{Si}$ atom had at the same site. This case has different structural and electronic characteristics than the rest of the substitutional sites: it has a shorter bond length for $\mathrm{S}$, at $2.17 \AA$, comparable to that of the interstitial sites, and it has an empty state in the gap, just below the minimum of the conduction band, again similar to the twofold coordinated interstitial sites. The substitutional site $\mathrm{T}_{7}^{(\mathrm{C})}$ also exhibits some special features: even though its threefold coordination implies the breaking of a covalent bond, the local geometry leads to the formation of a nearby fivefold coordinated $\mathrm{Si}$ atom, which introduces two states in the band gap, an occupied deep gap state (at $0.34 \mathrm{eV}$ ) related to the Si coordination defect and an empty gap state just below the conduction band minimum (at $0.95 \mathrm{eV}$ ) related to $\mathrm{S}$.

Judging from these results, we can infer that essentially in all cases, the substitutional $\mathrm{S}$ atom will end up in a threefold coordinated site. Looking at the electronic properties of these 
TABLE IV. Energetics, geometric features and electronic structure of S subsitutional and interstitial dopants in amorphous Si sample C. The meaning of symbols is the same as in Table III. For the interstitial cases $\mathrm{I}_{\mathrm{n}}^{(\mathrm{C})}, n=1-3, \bar{b}_{i}$ denotes the $\mathrm{Si}-\mathrm{Si}$ bond length into which the $\mathrm{S}$ atom is introduced and $\bar{b}_{f}$ denotes the average bond length of the $\mathrm{S}$ atom after relaxation. $\varepsilon_{d}$ is the position of the dopant state in the gap relative to the valence band maximum and $\varepsilon_{F}$ is the position of the Fermi level. The energy gap of the pure amorphous Si sample is $1.05 \mathrm{eV}$ and its Fermi level is at $0.01 \mathrm{eV}$.

\begin{tabular}{cccccccc}
\hline \hline Site & $E_{f}$ & $\bar{b}_{i}$ & $C_{f}$ & $\bar{b}_{f}$ & $\varepsilon_{\text {gap }}$ & $\varepsilon_{d}$ & $\varepsilon_{F}$ \\
\hline $\mathrm{I}_{1}^{(\mathrm{C})}$ & -5.14 & 2.44 & 2 & 2.14 & 1.01 & 0.96 & 0.02 \\
$\mathrm{I}_{2}^{(\mathrm{C})}$ & -5.03 & 2.48 & 2 & 2.14 & 1.05 & & 0.03 \\
$\mathrm{I}_{3}^{(\mathrm{C})}$ & -5.01 & 2.48 & 2 & 2.14 & 1.02 & & 0.01 \\
$\mathrm{~T}_{6}^{(\mathrm{C})}$ & -4.56 & 2.39 & 2 & 2.17 & 1.10 & 0.91 & 0.01 \\
\hline $\mathrm{T}_{7}^{(\mathrm{C})}$ & -4.74 & 2.31 & 3 & 2.26 & 1.05 & $0.34,0.95$ & 0.34 \\
$\mathrm{~T}_{8}^{(\mathrm{C})}$ & -4.35 & 2.38 & 3 & 2.27 & 1.05 & 0.36 & 0.38 \\
$\mathrm{~T}_{9}^{(\mathrm{C})}$ & -4.15 & 2.33 & 3 & 2.23 & 1.08 & 0.47 & 0.49 \\
$\mathrm{~T}_{10}^{(\mathrm{C})}$ & -3.85 & 2.33 & 3 & 2.28 & 1.06 & 0.80 & 0.83 \\
$\mathrm{~T}_{11}^{(\mathrm{C})}$ & -3.63 & 2.34 & 3 & 2.30 & 1.04 & 0.49 & 0.49 \\
$\mathrm{~T}_{12}^{(\mathrm{C})}$ & -3.52 & 2.35 & 3 & 2.31 & 1.07 & 0.60 & 0.62 \\
\hline $\mathrm{T}_{13}^{(\mathrm{C})}$ & -3.89 & 2.30 & 4 & 2.32 & 1.07 & 0.89 & 0.91 \\
\hline \hline
\end{tabular}

samples, we find that their Fermi energies are shifted up by roughly $0.5 \mathrm{eV}$, compared to the original Fermi level for the pure sample C. Each of them has a doubly filled defect state near the middle of the gap. Detailed analysis by projecting electronic wave functions of the sample onto $s, p$, and $d$ spherical harmonics centered at the positions of the ions, shows that these gap states are mainly localized on the neighboring $\mathrm{Si}$ atom that has the longest bond length from the substitutional $\mathrm{S}$ atom. These observations fit well with the model built by Street for substitutional defects in amorphous silicon, ${ }^{52}$ in the context of which we analyze next the behavior of the $\mathrm{S}$ dopants.

According to Mott's 8-N rule, a neutral $\mathrm{S}$ atom would have to form two covalent bonds and be in state $S_{2}^{0}$. This strict rule forbids substitutional doping in amorphous semiconductors. But experimental results show that low level doping actually does occur in amorphous semiconductors. In our case, as in experiment, ${ }^{22}$ the doping level of $\mathrm{S}$ is about $1 \%$. Street ${ }^{52}$ relaxed the $8-\mathrm{N}$ rule by allowing the doping atoms to be ionized. This model, applied to our results yields the following picture: by losing one electron, the $S$ atom turns into a threefold coordinated state, $\mathrm{S}_{3}^{+}$, while at the same time a neighboring $\mathrm{Si}$ atom accepts the electron and forms a threefolded coordinated dangling bond state $\mathrm{D}^{-}$(referred to as compensating state ${ }^{52}$ ). The antibonding state of $\mathrm{S}_{3}^{+}$is empty but the $\mathrm{D}^{-}$state in the gap is filled. This is why the position of the Fermi level in these cases is shifted up by about $0.5 \mathrm{eV}$. From Table IV, a higher shift of the Fermi level corresponds to a higher formation energy. It is also clear that high level doping is not possible because the Fermi level can only shift up to the lowest conduction band.

When there is not enough room for complete relaxation, as in case $\mathrm{T}_{13}^{(\mathrm{C})}$, where the $\mathrm{Si}$ atom had the smallest average bond length in the original configuration at $2.30 \AA$, the $\mathrm{S}$ atom may be in a nominally fourfold coordination but actu- ally with only three close neighbors. In this case a $\mathrm{S}_{4}^{0}$ state is formed. Two extra electrons that are not in bonds will occupy the antibonding state. This is why the Fermi level of this configuration is shifted up by about $1.0 \mathrm{eV}$, and ends up just below the conduction band and higher than the deep gap states associated with $\mathrm{D}^{-}$.

Last, we re-examine the $\mathrm{S}$ substitutional case labeled $\mathrm{T}_{6}^{(\mathrm{C})}$, in which the $\mathrm{S}$ atom is twofold coordinated. In this case there is no shift in the Fermi level and the defect state below the conduction band is empty. This configuration also corresponds to relatively low formation energy. This raises the question of whether volume restrictions lead to creation of a majority of threefold coordinated sites for S, as opposed to twofold coordinated sites that seem to be a more natural state for this impurity. In order to address this question, we examined carefully the bond lengths of the three $\mathrm{S}-\mathrm{Si}$ bonds for each threefold coordinated $\mathrm{S}$ atom and found that, unlike the situation in $\mathrm{T}_{13}^{(\mathrm{C})}$, these bonds have essentially equal lengths. There is no obvious trend for breaking any one of them. Thus we conclude that the electronic properties must be playing a more important role. Specifically, a change from the $\mathrm{S}_{3}^{+}$state back to the $S_{2}^{0}$ state requires transfering one electron to the $S$ atom while breaking a $\mathrm{S}-\mathrm{Si}$ bond and creating a new $\mathrm{D}^{-}$ state in the adjacent $\mathrm{Si}$ atom whose bond to $\mathrm{S}$ is broken. However, this coordination for the Si neighbor is forbidden by the $8-\mathrm{N}$ rule. Thus, although the twofold coordinated state for the $\mathrm{S}$ dopant would be preferred, because of the initial coordination number and the restrictions imposed by the $8-\mathrm{N}$ rule, threefold coordination is the most likely outcome.

For the amorphous samples A and B, it is hard to make a similar detailed analysis because of the existence of intrinsic coordination defects and corresponding defect states in the gap of these two samples. Nevertheless, the same explanation for the overwhelming threefold coordination of the $\mathrm{S}$ dopant atoms should still be valid. 


\section{CONCLUSION}

Our study of $\mathrm{S}$ doping of crystalline and amorphous $\mathrm{Si}$ samples has revealed a number of unexpected results, which we summarize here. In crystalline Si, we find that the substitutional position for the single $\mathrm{S}$ dopant atom is preferred. An interstitial $\mathrm{S}$ atom with a buckling angle $122^{\circ}$ has an energy $0.3 \mathrm{eV}$ higher than the substitutional one but is $0.95 \mathrm{eV}$ lower than a bond-center $\mathrm{S}$ atom without buckling. Two $\mathrm{S}$ dopant atoms form a nearest-neighbor substitutional pair which has an energy lower than the two isolated substitutional $\mathrm{S}$ atoms by $1.53 \mathrm{eV}$, even though the two $\mathrm{S}$ atoms in the pair are not bonded covalently and each one is threefold coordinated. In coordination-defect-free amorphous $\mathrm{Si}$, we found that interstitial twofold coordinated $\mathrm{S}$ atoms have the lowest formation energy. Substitutional S atoms, in both the coordination-defect-free sample and the defective samples, tend to form structures of mostly threefold coordination. This is expected when the original $\mathrm{Si}$ site is a dangling bond coordination defect, but it is also true when the original site is fourfold or even fivefold coordinated (a floating bond defect). Exceptions occur in special cases, when the local environment allows the substitutional $\mathrm{S}$ atom to break one or two bonds and become twofold coordinated, while the neighboring Si atoms relax without introducing a high energy cost. Thus, we conclude that under equilibrium conditions the overwhelming majority of substituional $\mathrm{S}$ atoms will be threefold coordinated, either by forming substitutional pairs in crystalline $\mathrm{Si}$, or by substituting $\mathrm{Si}$ atoms in the most common sites (fourfold coordinated or dangling bonds) in amorphous $\mathrm{Si}$, a conclusion supported by a detailed analysis of the electronic properties.

We can now use the results of this study of S point defects in crystalline and amorphous Si to interpret the experimental findings related to the so called "black Si." 22 In preparing these samples, the eperimenters used nanosecond (ns) and femtosecond (fs) laser irradiation. The ns-irradiated structure has very little disorder while the fs-irradiated sample is covered by a disordered nanocrystalline surface layer. An amorphous layer is created on both samples, in which $\mathrm{S}$ atoms are incorporated. These $\mathrm{S}$ atoms, according to our study, will mostly be threefold coordinated. The presence of intrinsic coordination defects (dangling and floating bonds) in the amorphous layers makes the samples light absorbing (black). After annealing, the ns-irradiated sample reverts to crystalline structure and $\mathrm{S}$ atoms are forced to be threefold or fourfold coordinated in the lowest energy substitutional positions. These $\mathrm{S}$ atoms lead to defect-induced occupied gap states. Thus, the ns-irradiated sample remains lightabsorbing, although the absorption of light is lower compared to the sample before annealing. On the other hand, the fs-irradiated sample is highly disordered. Annealing leads to elimination of some of its intrinsic defects and rearrange- ment of S atoms, but not complete crystallization. According to the estimation made by Roorda and Sinke, ${ }^{53}$ after annealing, the remaining concentration of coordination defects in fs-irradiated sample will be about $1 \%$. During annealing, we expect the $\mathrm{S}$ atoms to saturate these defects. Furthermore, sufficient annealing should allow the $\mathrm{S}$ atoms to become twofold coordinated, because the lowest energy sample with defects we have found is sample $\mathrm{A}$, in which a S substitutional atom at what was originally a dangling bond defect actually becomes twofold coordinated with a relatively low formation energy [case $\mathrm{D}_{4}^{(\mathrm{A})}$ in Table III]. Thus, we expect to recover a band gap devoid of defect states in the fs-irradiated sample, with the sample becoming transparent again, as is indeed observed in experiment.

Finally, our study affords some theoretical predictions about the behavior of $\mathrm{S}$-doped $\mathrm{Si}$, under equilibrium conditions: In crystalline silicon samples, the $\mathrm{S}$ atoms will be preferentially at substitutional sites and thus they will introduce occupied states deep inside the band gap, which should make the sample light absorbing. Annealing of the sample will create S-substitutional pairs if the kinetic barriers for this process are not prohibitively high, but this will not change the optical properties. If larger clusters of $\mathrm{S}$ atoms can be created, then the optical properties may change depending on the geometry and coordination of $\mathrm{S}$ atoms in the cluster. In amorphous silicon, the $\mathrm{S}$ atoms originally will either be placed at interstitial sites, which requires small changes in the structure and keeps the intrinsic defects of the samples, or they will be substitutional at sites where the intrinsic coordination defects were. Sufficient amount of annealing without crystallization, is likely to lead to the energetically favored two-fold coordination state for $\mathrm{S}$, which achieves two effects: it eliminates the coordination-induced defect states in the gap and it does not introduce any dopant-related states in the gap. If the level of doping is sufficient to compensate for all the coordination defects, then the sample will develop a gap in the electronic spectrum and become transparent to the corresponding range of radiation.

Following this detailed analysis of the geometric and electronic structure of equilibrium configurations for $\mathrm{S}$ dopants in crystalline and amorphous $\mathrm{Si}$, it would be of great interest to study the kinetics of $\mathrm{S}$ incorporation in bulk $\mathrm{Si}$ to determine whether or not kinetic barriers play an important role in what is actually observed experimentally.

\section{ACKNOWLEDGMENTS}

The authors thank Dr. Zhenyu Zhang and Dr. Gang Lu for many helpful discussions. They are particularly indebted to Prof. M. J. Aziz for many helpful discussions and clarification of the experimental results. The work of E.K. is in part supported by the Nanoscale Science and Engineering Center of Harvard University, which is funded by the National Science Foundation. 
${ }^{1}$ J. Coutinho, R. Jones, P. R. Briddon, and S. Öberg, Phys. Rev. B 62, 10824 (2000).

${ }^{2}$ M. Pesola, J. von Boehm, T. Mattila, and R. M. Nieminen, Phys. Rev. B 60, 11449 (1999).

${ }^{3}$ A. B. Filonov, S. Ossicini, E. Bassani, and F. A. d'Avitaya, Phys. Rev. B 65, 195317 (2002).

${ }^{4}$ S. Öberg, C. P. Ewels, R. Jones, T. Hallberg, J. L. Lindström, L. I. Murin, and P. R. Briddon, Phys. Rev. Lett. 812930 (1998).

${ }^{5}$ See E. Kaxiras, Atomic and Electronic Structure of Solids (Cambridge University Press, Cambridge, 2003).

${ }^{6}$ G. Kim and J. D. Dow, Phys. Rev. B 40, 7888 (1989).

${ }^{7}$ H. Overhof, M. Scheffler, and C. M. Weinert, Phys. Rev. B 43, 12494 (1991).

${ }^{8}$ J. Coutinho, V. J. B. Torres, R. Jones, and P. R. Briddon, Phys. Rev. B 67, 035205 (2003).

${ }^{9}$ S. T. Pantelides, Phys. Rev. Lett. 57, 2979 (1986); 58, 1344 (1987).

${ }^{10}$ M. Durandurdu, D. A. Drabold, and N. Mousseau, Phys. Rev. B 62, 15307 (2000).

${ }^{11}$ J. Dong and D. A. Drabold, Phys. Rev. Lett. 80, 1928 (1998).

${ }^{12}$ J. H. Stathis and S. T. Pantelides, Phys. Rev. B 37, 6579 (1988).

${ }^{13}$ D. Redfield and R. H. Bube, Phys. Rev. Lett. 65, 464 (1990).

${ }^{14}$ R. Biswas, C. Z. Wang, C. T. Chan, K. M. Ho, and C. M. Soukoulis, Phys. Rev. Lett. 63, 1491 (1989).

${ }^{15}$ F. Valiquette and N. Mousseau, Phys. Rev. B 68, 125209 (2003).

${ }^{16}$ B. Tuttle and J. B. Adams, Phys. Rev. B 57, 12859 (1998).

${ }^{17}$ T. H. Her, R. J. Finlay, C. Wu, S. Deliwala, and E. Mazur, Appl. Phys. Lett. 73, 1673 (1998).

${ }^{18}$ A. J. Pedraza, J. D. Fowlkes, and D. H. Lowndes, Appl. Phys. Lett. 74, 2322 (1999).

${ }^{19}$ C. Wu, C. H. Crouch, L. Zhao, J. E. Carey, R. Younkin, J. A. Levinson, E. Mazur, R. M. Farrel, P. Gothoskar, and A. Karger, Appl. Phys. Lett. 78, 1850 (2001).

${ }^{20}$ J. E. Carey and E. Mazur (unpublished).

${ }^{21}$ C. H. Crouch, J. E. Carey, M. Y. Shen, E. Mazur, and F. Génin (unpublished).

${ }^{22}$ C. H. Crouch, J. E. Carey, J. M. Warrender, M. J. Aziz, and E. Mazur, Appl. Phys. Lett. 84, 1850 (2004).

${ }^{23}$ P. Hohenberg and W. Kohn, Phys. Rev. 136, B864 (1964).

${ }^{24}$ W. Kohn and L. Sham, Phys. Rev. 140, A1133 (1965).

${ }^{25}$ O. Gunnarsson and B. I. Lundqvist, Phys. Rev. B 13, 4247 (1976).

${ }^{26}$ J. P. Perdew, D. C. Langreth, and V. Shahni, Phys. Rev. Lett. 38, 1030 (1977).

${ }^{27}$ G. Kresse and J. Hafner, Phys. Rev. B 47, 558 (1993); 49, 14251 (1994); G. Kresse and J. Furthmüller, Comput. Mater. Sci. 6, 15 (1996); Phys. Rev. B 54, 11169 (1996).

${ }^{28}$ D. Vanderbilt, Phys. Rev. B 41, 7892 (1990); A. Pasquarello, K. Laasonen, R. Car, C. Lee, and D. Vanderbilt, Phys. Rev. Lett. 69, 1982 (1992); K. Lassonen, A. Pasquarello, R. Car, C. Lee, and D. Vanderbilt, Phys. Rev. B 47, 10142 (1993); G. Kresse and J. Hafner, J. Phys.: Condens. Matter 6, 8245 (1994).

${ }^{29}$ R. Car and M. Parrinello, Phys. Rev. Lett. 60, 204 (1998).

${ }^{30}$ I. Stich, R. Car, and M. Parrinello, Phys. Rev. B 44, 11092 (1991).

${ }^{31}$ H. Balamane, T. Halicioglu, and W. A. Tiller, Phys. Rev. B 46, 2250 (1992), and references therein.

${ }^{32}$ W. D. Luedtke and U. Landman, Phys. Rev. B 37, 4656 (1988).

${ }^{33}$ W. D. Luedtke and U. Landman, Phys. Rev. B 40, 1164 (1989).

${ }^{34}$ F. H. Stillinger and T. A. Weber, Phys. Rev. B 31, 5262 (1985).

${ }^{35}$ M. Ishimaru, M. Shinji, and T. Motooka, Phys. Rev. B 56, 15133 (1997).

${ }^{36}$ J. Tersoff, Phys. Rev. B 38, 9902 (1988); ibid. 39, 5566 (1989).

${ }^{37}$ F. Wooden, K. Winer, and D. Weaire, Phys. Rev. Lett. 54, 1392 (1985).

${ }^{38}$ F. Wooten and D. Weaire, Solid State Phys. 40, 1 (1987); B. R. Djordjević, M. F. Thorpe, and F. Wooden, Phys. Rev. B 52, 5685 (1995); G. T. Barkema, and N. Mousseau, ibid. 62, 4985 (2000).

${ }^{39}$ J. Fortner and J. S. Lannin, Phys. Rev. B 39, 5527 (1989).

${ }^{40}$ S. Kugler, G. Molnár, G. Petö, E. Zsoldos, L. Rosta, A. Menelle, and R. Bellissent, Phys. Rev. B 40, 8030 (1989).

${ }^{41}$ W. A. Kamitakahara, H. R. Shank, J. F. McClelland, U. Buchenau, F. Compf, and L. Pintschovons, Phys. Rev. Lett. 52, 644 (1984).

${ }^{42}$ M. Z. Bazant, E. Kaxiras, and J. F. Justo, Phys. Rev. B 56, 8542 (1997); M. Z. Bazant and E. Kaxiras, Phys. Rev. Lett. 77, 4370 (1996); M. Z. Bazant and E. Kaxiras, Mater. Res. Soc. Symp. Proc. 408, 79 (1996).

${ }^{43}$ J. F. Justo, M. Z. Bazant, E. Kaxiras, V. V. Bulatov, and S. Yip, Phys. Rev. B 58, 2539 (1998); M. Z. Bazant, E. Kaxiras, and J. F. Justo, Mater. Res. Soc. Symp. Proc. 491, 339 (1998).

${ }^{44}$ L. Brambilla, L. Colombo, V. Rosato, and F. Cleri, Appl. Phys. Lett. 77, 2337 (2000).

${ }^{45}$ P. Keblinski, M. Z. Bazant, R. K. Dash, and M. M. Treacy, Phys. Rev. B 66, 064104 (2002).

${ }^{46}$ C. L. Allred, M. Z. Bazant, and L. Hobbs (unpublished).

${ }^{47}$ M. O. Thompson, J. W. Mayer, A. G. Cullis, H. C. Weber, N. G. Chew, J. M. Poate, and D. C. Jackson, Phys. Rev. Lett. 50, 896 (1983); A. G. Cullis, N. G. Chew, H. C. Weber, and D. J. Smith, J. Cryst. Growth 68, 624 (1984).

${ }^{48}$ S. Roorda, W. C. Sinke, J. M. Poate, D. C. Jacobson, S. Dierker, B. S. Dennis, D. J. Eaglesham, F. Spaepen, and P. Fuoss, Phys. Rev. B 44, 3702 (1991).

${ }^{49}$ N. Fukata, A. Kasuya, and M. Suezawa, Physica B 308, 1125 (2001).

${ }^{50}$ M. S. Hybertsen and S. G. Louie, Phys. Rev. Lett. 55, 1418 (1985); Phys. Rev. B 34, 5390 (1986).

${ }^{51}$ R. W. Godby, M. Schlüter, and L. J. Sham, Phys. Rev. Lett. 56, 2415 (1986); Phys. Rev. B 37, 10159 (1988).

${ }^{52}$ R. A. Street, Phys. Rev. Lett. 49, 1187 (1982).

${ }^{53}$ S. Roorda and W. C. Sinke, Phys. Rev. B 44, 3702 (1991). 